\title{
RESEARCH
}

Open Access

\section{DLA class II risk haplotypes for autoimmune diseases in the bearded collie offer insight to autoimmunity signatures across dog breeds}

Liza C. Gershony ${ }^{1,2}$ (D), Janelle M. Belanger ${ }^{1}$, Andrea D. Short ${ }^{3}$, Myly Le ${ }^{1}$, Marjo K. Hytönen ${ }^{4,5}$, Hannes Lohi' ${ }^{4,5}$, Thomas R. Famula', Lorna J. Kennedy ${ }^{3}$ and Anita M. Oberbauer ${ }^{1 *}$

\begin{abstract}
Background: Primary hypoadrenocorticism (Addison's disease, AD) and symmetrical lupoid onychodystrophy (SLO) are two clinical conditions with an autoimmune etiology that occur in multiple dog breeds. In man, autoimmunity is associated with polymorphisms in immune-related genes that result in a reduced threshold for, or defective regulation of, T cell activation. The major histocompatibility complex (MHC) class II genes encode molecules that participate in these functions, and polymorphisms within these genes have been associated with autoimmune conditions in dogs and humans. Bearded collies have a relatively high prevalence of autoimmune diseases, particularly AD and SLO. Our study assessed the relationship between particular MHC (dog leukocyte antigen, DLA) class II haplotypes and the two autoimmune diseases most common in this breed. Moreover, five unrelated breeds at increased risk for AD were studied for comparative purposes and analyzed in the context of extant literature.

Results: A single DLA class II three-locus haplotype, determined by sequence-based typing, was associated with increased risk for $A D(D L A-D R B 1 * 009: 01 / D Q A 1 * 001: 01 / D Q B 1 * 008: 02)$ in bearded collies. Comparative analysis with the five additional breeds showed limited allele sharing, with DQA1*001:01 and DQB1*002:01 being the only alleles observed in all breeds. A distinct three-locus risk haplotype (DLA-DRB1*001:01/DQA1*001:01/DQB1*002:01) was associated with AD in the West Highland white terrier and Leonberger. Two different risk haplotypes were associated with increased risk for SLO in the bearded collie (DLA-DRB1*018:01/DQA1*001:01/DQB1*002:01 and DLADRB1*018:01/DQA 1*001:01/ DQB1*008:02).

Conclusion: Two-locus DQ haplotypes composed of DLA-DQA1*001:01 in association with DLA-DQB1*002:01 or DLA-DQB1*008:02 make up the four risk haplotypes identified in the present study and are also found in other risk haplotypes previously associated with diabetes mellitus and hypothyroidism across different dog breeds. Our findings build upon previously published data to suggest that this two-locus (DQ) model serves as a good indicator for susceptibility to multiple organ-specific autoimmune diseases in the canine population. However, it is also clear that additional loci are necessary for actual disease expression. Investigation of affected and unaffected dogs carrying these predisposing DQ haplotype signatures may allow for the identification of those additional genetic components that determine autoimmune disease expression and organ specificity.
\end{abstract}

Keywords: (3-10): Dog, Autoimmune, MHC, DLA, Addison's disease, SLO

\footnotetext{
* Correspondence: amoberbauer@ucdavis.edu

'Department of Animal Science, University of California, One Shields Ave,

Davis, CA 95616, USA

Full list of author information is available at the end of the article
}

(c) The Author(s). 2019 Open Access This article is distributed under the terms of the Creative Commons Attribution 4.0 International License (http://creativecommons.org/licenses/by/4.0/), which permits unrestricted use, distribution, and reproduction in any medium, provided you give appropriate credit to the original author(s) and the source, provide a link to the Creative Commons license, and indicate if changes were made. The Creative Commons Public Domain Dedication waiver (http://creativecommons.org/publicdomain/zero/1.0/) applies to the data made available in this article, unless otherwise stated. 


\section{Plain english summary}

Primary hypoadrenocorticism, also known as Addison's disease (AD), and symmetrical lupoid onychodystrophy (SLO) are two autoimmune conditions that occur in multiple dog breeds. Disease expression depends on a combination of genetic and environmental factors, and discovery of genetic loci involved in disease susceptibility may help understand and predict risk for disease. Autoimmunity in humans is associated with altered immune-related genes that result in defective regulation of the immune system. The strongest associations for many human autoimmune diseases involve the major histocompatibility complex (MHC) class II genes. Genetic variants within these genes have also been associated with autoimmune conditions in dogs. Bearded collies have a relatively high prevalence of autoimmune diseases, and our study assessed the relationship between particular MHC (dog leukocyte antigen, DLA) class II haplotypes and the two autoimmune diseases most common in this breed, $\mathrm{AD}$ and SLO. We also studied five unrelated dog breeds at high risk for $\mathrm{AD}$ to determine if there were haplotypes common across affected dogs in these breeds and bearded collies with AD. A single DLA class II three-locus haplotype was associated with increased risk for $\mathrm{AD}$ in bearded collies with a different three-locus risk haplotype associated with $\mathrm{AD}$ in the West Highland white terrier and Leonberger. Two separate three-locus risk haplotypes were associated with increased risk for SLO in the bearded collie. Importantly, two-locus DQ haplotypes composed of DLA-DQA1*001:01 in association with DLA-DQB1*002:01 or DLA-DQB1*008:02 were common across the breeds and autoimmune conditions, and made up the four risk haplotypes identified in the present study. In the published literature, these same two-locus haplotypes are also found in risk haplotypes associated with diabetes mellitus and hypothyroidism across different dog breeds, suggesting that this two-locus model serves as a good indicator for susceptibility to multiple organ-specific autoimmune diseases in the dog population. However, many dogs carrying these haplotypes never develop clinical autoimmune disease, making it clear that additional genes are necessary for actual disease expression. Investigation of affected and unaffected dogs carrying these predisposing DQ haplotype signatures may allow for the identification of those additional genetic components that determine autoimmune disease expression and organ specificity.

\section{Background}

Primary hypoadrenocorticism (Addison's disease, AD) is a life-threatening clinical condition in dogs characterized by inadequate secretion of adrenocortical hormones by the adrenal glands [1-5]. In both humans and dogs, this endocrine disorder is caused by gradual immune-mediated destruction of the adrenal cortex [1,4-7]. The presence of autoantibodies against adrenal antigens detected in both human $[8,9]$ and canine patients [2] with naturally occurring $\mathrm{AD}$ provide further evidence of the immune-mediated etiopathogenesis of $\mathrm{AD}$. $\mathrm{AD}$ has been reported in many purebred and mixed breed dogs (OMIA 000519-9615) [1, $10,11]$ with disease prevalence ranging from 0.06 to $0.4 \%$ in the overall dog population [12-15]. However, within certain breeds, prevalence of $\mathrm{AD}$ can be as high as $9 \%[7,10$, $16,17]$. Reported breeds at increased risk for developing AD include bearded collies, Portuguese water dogs (PWD), standard poodles, West Highland white terriers (WHWT), Leonbergers, Wheaten terriers and Nova Scotia duck tolling retrievers $[1,7,10,14,16-20]$.

Symmetrical lupoid onychodystrophy (SLO) is another condition of autoimmune etiology that affects multiple dog breeds, such as the bearded collie, Gordon setter, English setter, giant schnauzer, Labrador retriever, Welsh corgi, boxer, and German shorthair pointer with variable prevalence (OMIA 001989-9615) [11, 21-25]. SLO is a clinical syndrome characterized by sloughing claws and secondary bacterial infection that was first described in 1992 [24, 26, 27]. Since then, most research has focused on disease diagnosis and treatment $[22,25]$. While little research exists on the cause of SLO, Wilbe et al. [24] and Dahlgren et al. [28] have reported an association with particular major histocompatibility complex (MHC; or Dog Leukocyte Antigen, DLA) class II alleles in bearded collies, giant schnauzers, and English and Gordon setters $[24,28]$. DLA class II haplotypes were also shown to be more prevalent among dogs of multiple breeds with $\mathrm{AD}$ [3].

Bearded collies, in particular, have a relatively high prevalence of autoimmune diseases. Health reports produced by the Bearded Collie Foundation for Health indicate $11.2 \%$ of registered dogs have one or more autoimmune disease [29]. Among these, more than half were diagnosed with AD or SLO. Autoimmune diseases such as AD and SLO are considered complex disorders that likely result from the combination of a predisposing genetic background and environmental factors [30]. The environmental triggers necessary or sufficient for autoimmune disease expression remain unclear, but discovery of the genetic loci involved in disease susceptibility may prove helpful in understanding and predicting risk for disease [31]. In humans, it is hypothesized that various polymorphisms in immune-related genes contribute to a reduced threshold for autoreactive lymphocyte activation and/or to defective regulation of autoreactive $\mathrm{T}$ cell responses [30]. Likely due to its role in recognition of self versus non-self, variation within MHC class II alleles has been implicated in multiple human autoimmune disorders including Addison's disease, type 1 diabetes mellitus and inflammatory bowel disease [30, 32-34]. Similar connections have been made for dogs, and review of the published literature shows that several 
autoimmune conditions are associated with DLA class II risk haplotypes (as shown in Table 1) [3, 24, 28, 35-45].

Given the relatively high prevalence of autoimmune diseases in bearded collies, the present study assessed the relationship between particular MHC class II haplotypes and the two autoimmune conditions commonly observed in this breed. As a corollary, those haplotypes were evaluated in five other dog breeds at high risk for $\mathrm{AD}$ to determine if there were common DLA haplotype signatures associated with the disease across multiple breeds. This is the largest DLA study on AD and SLO bearded collies.

\section{Results}

Two hundred and thirty-six bearded collies were haplotyped for the three polymorphic DLA class II genes: 125 healthy dogs ( 57 males, 68 females), $61 \mathrm{AD}$ ( 22 males and 39 females) and 50 SLO (26 males, 24 females). Quality sequences of all three genes could not be obtained for three male control dogs, which were removed from further analysis.

Within the study population, six DLA-DRB1 alleles were identified, three of which were uncommon and only observed in healthy control dogs (allele frequency < 3\%; Additional file 1: Table S1). Four DLA-DQA1 alleles were identified, two of which were only seen in a small number of controls (allele frequency $<3 \%$ ). Similarly, seven DLA-DQB1 alleles were identified, three of which were less frequent (two found in control dogs only, and one in both $\mathrm{AD}$ and control dogs). Among the nine three-locus haplotypes identified (coded 1 through 9 for ease in presentation) in the bearded collie study population, four were infrequent in the breed (Table 2).

\section{$A D$ in bearded collies}

Three DLA-DRB1 alleles (DLA-DRB1*009:01, DLADRB1*015:01 and DLA-DRB1*018:01) were frequent among bearded collie AD cases; DLA-DRB1*009:01 was the only allele associated with higher risk for $\mathrm{AD}$

Table 1 Published three-locus DLA class II haplotypes associated with increased risk for some immune-related diseases in dogs

\begin{tabular}{|c|c|c|c|c|c|}
\hline Disease & DLA-DRB1 & DLA-DQA1 & DLA-DQB1 & Breed(s) & Reference \\
\hline \multirow[t]{7}{*}{$A D$} & 009:01 & 001:01 & 008:02 & Bearded collie & \multirow[t]{6}{*}{ Massey et al. 2013 [3] } \\
\hline & 009:01 & 001:01 & 008:01:1 & Cocker spaniel & \\
\hline & 015:01 & 006:01 & 023:01 & Cocker spaniel, Springer spaniel & \\
\hline & 001:01 & 002:01 & 013:03 & Springer spaniel & \\
\hline & 001:01 & 001:01 & 002:01 & Labrador retriever, WHWT & \\
\hline & 015:02 & 006:01 & 023:01 & Standard poodle & \\
\hline & 015:02 & 006:01 & 023:01 & NSDTR & Hughes et al. 2010 [38] \\
\hline \multirow[t]{4}{*}{ SLO } & 018:01 & 001:01 & 008:02 & Bearded collie, Gordon setter & $\begin{array}{l}\text { Wilbe et al. 2010; Ziener et al. } \\
2015[24,43]\end{array}$ \\
\hline & 018:01 & 001:01 & 002:01 & Bearded collie & \multirow{2}{*}{$\begin{array}{l}\text { Wilbe et al. 2010; Dahlgren et al. } \\
2016[24,28]\end{array}$} \\
\hline & 001:01 & 001:01 & 002:01 & Giant schnauzer, English setter & \\
\hline & $101: 02^{\mathrm{a}}$ & 001:01 & 002:01 & English setter & Dahlgren et al. 2016 [28] \\
\hline \multirow[t]{5}{*}{ Hypothyroidism } & 012:01 & 001:01 & 002:01 & Doberman pinscher, Giant schnauzer & $\begin{array}{l}\text { Kennedy et al. 2006; Wilbe et al. } \\
2010[40,44]\end{array}$ \\
\hline & 001:07 & 001:01 & 002:01 & English setter & \multirow[t]{4}{*}{ Ziener et al. 2015 [43] } \\
\hline & 001:03 & 001:01 & 002:01 & Gordon setter & \\
\hline & 001:01 & 001:01 & 002:01 & & \\
\hline & 049:01 & 010:01 & 019:01 & & \\
\hline \multirow[t]{3}{*}{ Diabetes mellitus } & 009 & 001 & 008 & Multiple breeds & \multirow[t]{3}{*}{ Kennedy et al. 2006 [39] } \\
\hline & 015 & 006 & 023 & & \\
\hline & 002 & 009 & 001 & & \\
\hline Hepatitis & 006:01 & 004:01 & 013:03 & Doberman pinscher & Dyggve et al. 2011 [36] \\
\hline Anal furunculosis & 001:01 & 001:01 & 002:01 & German shepherd & Kennedy et al. 2008 [42] \\
\hline Necrotizing meningoencephalitis & 010:01:1 & 002:01 & 015:01 & Pug & Greer et al. 2010 [37] \\
\hline \multirow[t]{2}{*}{ Meningoencephalitis } & 018:02 & 001:01 & 008:02 & Greyhound & \multirow[t]{2}{*}{ Shiel et al. 2014 [45] } \\
\hline & 015:01 & 006:01 & 022:01 & & \\
\hline
\end{tabular}


Table 2 Frequency, code, and odds ratio (OR) with 95\% confidence interval (Cl) of each three-locus haplotype observed in healthy, Addisonian (AD) and symmetrical lupoid onychodystrophy (SLO) bearded collies

\begin{tabular}{|c|c|c|c|c|c|c|c|c|c|c|c|}
\hline \multirow[t]{2}{*}{ Code } & \multirow{2}{*}{$\begin{array}{l}\text { Haplotype } \\
\text { DLA-DRB1/DQA1/DQB1 }\end{array}$} & \multicolumn{2}{|l|}{ Controls } & \multicolumn{4}{|l|}{$A D$} & \multicolumn{4}{|l|}{ SLO } \\
\hline & & $2 n=244$ & $\%$ & $2 n=122$ & $\%$ & OR $(95 \% \mathrm{Cl})$ & $p$-value ${ }^{\dagger}$ & $2 n=100$ & $\%$ & OR $(95 \% \mathrm{Cl})$ & $p$-value \\
\hline 1 & 009:01/001:01/008:02 & 24 & 9.8 & 25 & 20.5 & $2.36(1.29-4.34)$ & 0.0058 & 1 & 1.0 & $0.09(0.01-0.70)$ & 0.0047 \\
\hline 2 & 015:01/006:01/003:01 & 31 & 12.7 & 18 & 14.8 & $1.19(0.64-2.22)$ & 0.6263 & 4 & 4.0 & $0.29(0.10-0.83)$ & 0.0172 \\
\hline 3 & 015:01/006:01/023:01 & 33 & 13.6 & 23 & 18.9 & $1.49(0.83-2.66)$ & 0.2177 & 2 & 2.0 & $0.13(0.03-0.55)$ & 0.0013 \\
\hline 4 & 018:01/001:01/002:01 & 73 & 29.9 & 27 & 22.1 & $0.67(0.40-1.11)$ & 0.1355 & 47 & 47.0 & $2.08(1.29-3.35)$ & 0.0029 \\
\hline 5 & 018:01/001:01/008:02 & 70 & 28.7 & 27 & 22.1 & $0.71(0.42-1.18)$ & 0.2094 & 46 & 46.0 & $2.12(1.31-3.43)$ & 0.0026 \\
\hline 6 & 015:01/006:01/022:01 & 5 & 2.0 & 2 & 1.6 & $0.80(0.15-4.17)$ & 1 & 0 & 0.0 & N/A & \\
\hline 7 & 002:01/009:01/001:01 & 6 & 2.5 & 0 & 0.0 & N/A & & 0 & 0.0 & N/A & \\
\hline 8 & 023:01/003:01/005:01 & 1 & 0.4 & 0 & 0.0 & N/A & & 0 & 0.0 & N/A & \\
\hline 9 & 015:02/006:01/023:01 & 1 & 0.4 & 0 & 0.0 & $\mathrm{~N} / \mathrm{A}$ & & 0 & 0.0 & N/A & \\
\hline
\end{tabular}

$N / A$ not enough data points to calculate; ${ }^{\dagger}$ Fisher's exact $p$-value

Text in bold indicates the haplotype frequency significantly differed between cases and controls

$(\mathrm{OR}=2.36, p=0.0058$; Additional file 2: Table S2). A single three-locus haplotype (haplotype 1), containing DLA-DRB1*009:01, was overrepresented among the AD dogs (Table 2). Increased risk for $\mathrm{AD}$ was observed in dogs that carried haplotype 1 although only two AD cases were homozygous for this haplotype. The majority of $\mathrm{AD}$ cases were heterozygous for haplotype $1(n=21)$, with half of those $(n=11)$ also carrying haplotype 5 . Bearded collies carrying the heterozygous genotype 15 were at higher risk for $\mathrm{AD}$ (Table 3). No differences in DLA homozygosity were noted when comparing AD dogs to controls (Table 4).

A subset of bearded collies $(n=219)$ that excluded those dogs carrying less frequent haplotypes (i.e., haplotypes $6-9 ; n=14$ ) was subjected to pairwise genotypic comparisons using logistic regression. A comparison between healthy and AD dogs showed genotypes 4 4, 4 5, and 55 were associated with the lowest probability for $\mathrm{AD}$ and genotypes that included haplotype 1 were associated with the highest probability (Fig. 1a). No sex differences were observed in the analysis $(p>0.05)$.
A second data set containing a total of 75 United Kingdom bearded collies (29 AD, 46 controls) included in a previously published study [3] was analyzed for comparison. Common haplotypes in the bearded collie breed (haplotypes 1-5) were seen in similar proportions in both data sets, and analyzing the merged data further corroborated the association between haplotype 1 and $\mathrm{AD} \quad(\mathrm{OR}=2.87 ; \quad 95 \% \quad \mathrm{CI}=1.76-4.66 ; \quad p=0.00002$; Additional file 3: Table S3). Six three-locus haplotypes observed in the published data (allele frequency $<3 \%$ ) were not seen in the new dataset. Conversely, haplotype 6 from the new dataset had not been observed in the published data. The combined dataset showed haplotype 4 underrepresented in AD dogs. To account for possible location influences, a geographical analysis of haplotype frequency in the combined dataset, which consisted predominantly of dogs from North America and Europe, showed differences in haplotype frequency between North American and European bearded collies (Additional file 4: Table S4). Haplotype 3 was more

Table 3 DLA class II genotypes significantly associated with Addison's disease (AD) and symmetrical lupoid onychodystrophy (SLO) in 233 Bearded collies, and corresponding odds ratios (OR) with the 95\% confidence interval (CI) using the DLA haplotype codes reported in Table 2

\begin{tabular}{|c|c|c|c|c|c|c|c|}
\hline \multirow[t]{2}{*}{ Disease } & \multirow[t]{2}{*}{ Genotypes } & \multicolumn{2}{|c|}{ Controls } & \multicolumn{2}{|c|}{ Cases } & \multirow[t]{2}{*}{$p$-value ${ }^{\dagger}$} & \multirow[t]{2}{*}{ OR $(95 \% \mathrm{Cl})$} \\
\hline & & $n$ & $\%$ & $n$ & $\%$ & & \\
\hline$A D$ & All genotypes containing haplotype 1 & 23 & 18.9 & 23 & 37.7 & 0.0070 & $2.6(1.31-5.19)$ \\
\hline$A D$ & Genotype 15 & 7 & 5.7 & 11 & 18.0 & 0.0154 & $3.6(1.32-9.87)$ \\
\hline SLO & All genotypes containing haplotype 4 & 62 & 50.8 & 36 & 72.0 & 0.0115 & $2.5(1.22-5.07)$ \\
\hline SLO & All genotypes containing haplotype 5 & 59 & 48.4 & 35 & 70.0 & 0.0114 & $2.5(1.24-5.02)$ \\
\hline SLO & Genotype 44 & 11 & 9.0 & 11 & 22.0 & 0.0258 & $2.8(1.14-7.08)$ \\
\hline SLO & Genotype 55 & 11 & 9.0 & 11 & 22.0 & 0.0258 & $2.8(1.14-7.08)$ \\
\hline SLO & Genotype 45 & 23 & 18.9 & 22 & 44.0 & 0.0011 & $3.4(1.65-6.94)$ \\
\hline SLO & Genotypes containing haplotypes 4 and/or 5 & 98 & 80.3 & 49 & 98.0 & 0.0033 & $12.0(1.58-91.33)$ \\
\hline
\end{tabular}

${ }^{\dagger}$ Fisher's exact $p$-value, significant at $p<0.05$ 
Table 4 Homozygosity across DLA class II genes in 233 Bearded collies (61 AD, 50 SLO and 122 controls). Different letters in the same column indicate statistical difference $(p<0.05)$ in DLA homozygosity across health status

\begin{tabular}{|c|c|c|c|c|c|c|c|c|}
\hline & \multicolumn{2}{|c|}{$\underline{\text { DLA-DRB1 }}$} & \multicolumn{2}{|c|}{ DLA-DQA1 } & \multicolumn{2}{|c|}{$\underline{\text { DLA-DQB1 }}$} & \multicolumn{2}{|c|}{$\underline{\text { DLA-DRB1/DQA1/DQB1 }}$} \\
\hline & $n$ & $\%$ & $n$ & $\%$ & $n$ & $\%$ & $n$ & $\%$ \\
\hline$A D$ & 26 & $42.6 \%^{a}$ & 45 & $73.8 \%^{\mathrm{a}}$ & 23 & $37.7 \%^{a}$ & 12 & $19.7 \%^{\mathrm{a}}$ \\
\hline SLO & 45 & $90.0 \%{ }^{b}$ & 46 & $92.0 \%{ }^{\mathrm{b}}$ & 23 & $46.0 \%^{\mathrm{a}}$ & 22 & $44.0 \%{ }^{b}$ \\
\hline Controls & 62 & $50.8 \%^{\mathrm{a}}$ & 79 & $64.8 \%{ }^{\mathrm{a}}$ & 36 & $29.5 \%^{\mathrm{a}}$ & 29 & $23.8 \%^{\mathrm{a}}$ \\
\hline
\end{tabular}

$n=$ number of homozygous dogs

$A D$ Addison's disease, SLO symmetrical lupoid onychodystrophy

prevalent among North American control and AD dogs, whereas haplotype 4 was more prevalent among European controls and haplotype 2 was more prevalent in North American AD dogs. Nevertheless, haplotype 1 remained associated with increased risk for $\mathrm{AD}$ in both regions (Additional file 5: Table S5). No significant differences were seen in haplotype frequency between the regions for SLO dogs. The combined dataset also included dogs from Australia (7 controls and 1 SLO) and New Zealand (2 controls, 2 AD and 1 SLO), however the low sample numbers precluded meaningful statistical comparisons for those regions.

\section{$A D$ in other breeds}

Seventeen DLA-DRB1 alleles were identified among all the breeds assessed, including the bearded collies (Additional file 6: Table S6). Seven of those were shared by three or more breeds whereas five were seen in only one of the breeds. The DLA-DRB1*008:02 and -DRB1*011:01 alleles were only seen in the PWD, DLA-DRB1*016:01 in the Leonberger, DLA-DRB1*017:01 in the WHWT and DLA-DRB1*084:01 in the Labradoodle. Among the seven DQA1 alleles, four were shared by three or more breeds. DLA-DQB1 was diverse across breeds, with 16 different alleles noted, only five of which were seen in more than two breeds. Despite the considerable diversity of alleles seen among the three polymorphic DLA class II genes across the six studied breeds at increased risk for AD, DLA-DQA1*001:01 and DQB1*002:01 were the only alleles shared by all six breeds.

Individual DLA class II alleles were significantly associated with AD in the WHWT (DLA-DQA1*001:01 and DLADQB1*002:01 - Additional file 7: Table S7) and Leonbergers (DLA-DRB1*001:01 - Additional file 8: Table S8). These alleles are a part of a three-locus haplotype that was significantly associated with AD status in WHWTs and Leonbergers (haplotype 14; Table 5). No alleles showed association with AD in the PWDs (Additional file 9: Table S9),
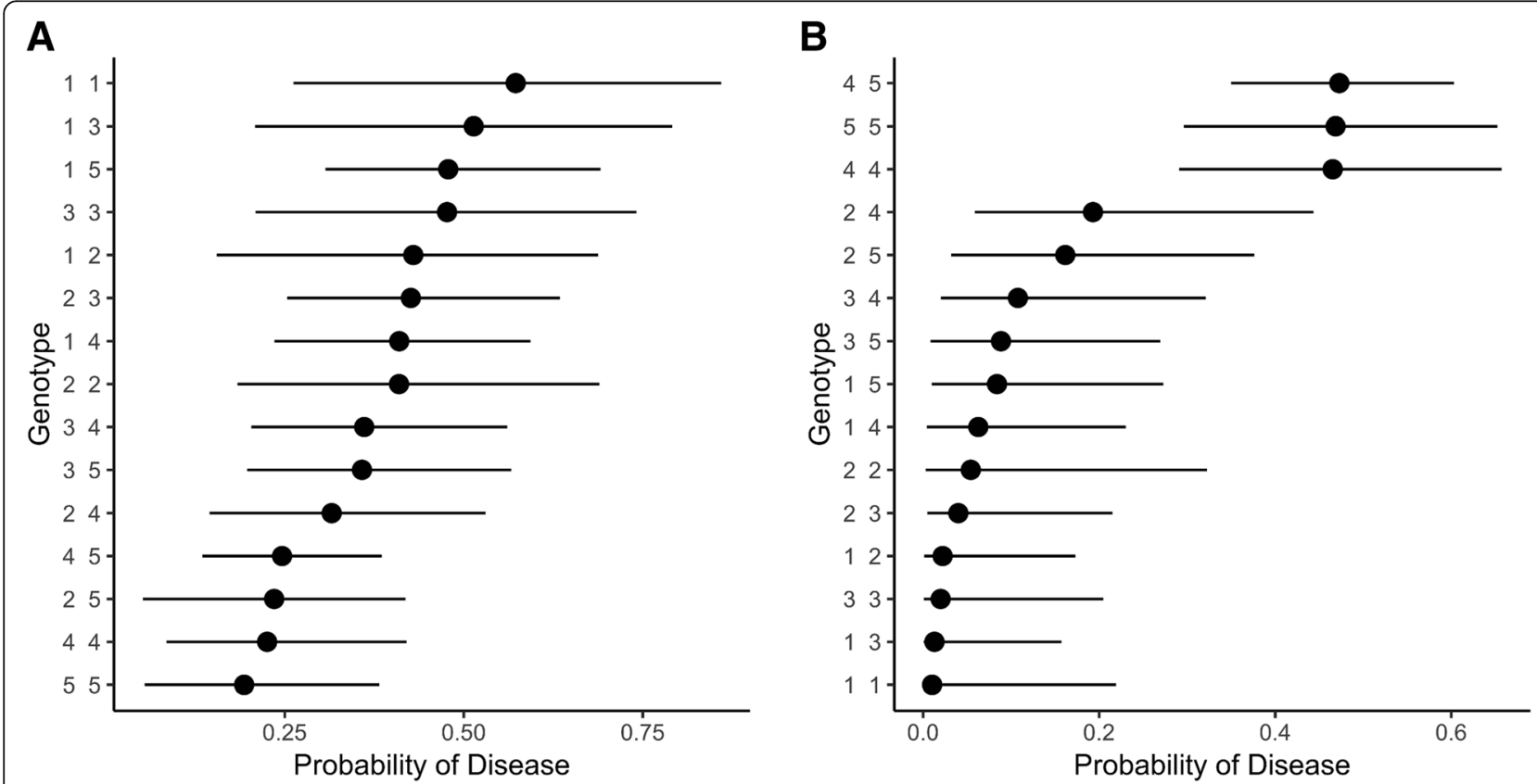

Fig. 1 Probability of (a) Addison's disease and (b) symmetrical lupoid onychodystrophy associated with the most common genotypic combinations of three-locus haplotypes observed in bearded collies $(n=219)$. Dots indicate the estimated probability of disease and lines represent the $95 \%$ confidence interval. DLA haplotype codes are as reported in Table 2 
Table 5 Frequency, code and odds ratio (OR) for DLA class II three-locus haplotypes observed in the six studied breeds at increased risk for Addison's disease (AD): bearded collie (61 cases, 122 controls), West Highland white terrier (WHWT; 43 cases, 166 controls), standard poodles (30 cases, 55 controls), Portuguese water dog (PWD; 17 cases, 76 controls), Labradoodle (12 cases, 9 controls) and Leonberger (11 cases, 14 controls)

\begin{tabular}{|c|c|c|c|c|c|c|c|c|c|}
\hline Code & DRB1 & DQA1 & DQB1 & Breed & $\begin{array}{l}\text { Control } \\
(2 n)\end{array}$ & $\begin{array}{l}\mathrm{AD} \\
(2 n)\end{array}$ & OR & $95 \% \mathrm{Cl}$ & $p$-value ${ }^{\dagger}$ \\
\hline 1 & 009:01 & 001:01 & 008:02 & Bearded collie & 24 & 25 & 2.36 & $1.29-4.34$ & 0.0058 \\
\hline 2 & 015:01 & 006:01 & 003:01 & Bearded collie & 31 & 18 & 1.19 & $0.64-2.22$ & 0.6263 \\
\hline \multirow[t]{4}{*}{3} & 015:01 & 006:01 & 023:01 & Bearded collie & 33 & 23 & 1.49 & $0.83-2.66$ & 0.2177 \\
\hline & & & & Labradoodle & 2 & 8 & 4.0 & $0.73-21.8$ & 0.1466 \\
\hline & & & & Standard poodle & 68 & 37 & 0.99 & $0.52-1.90$ & 1 \\
\hline & & & & WHWT & 17 & 0 & N/A & & \\
\hline \multirow[t]{2}{*}{4} & 018:01 & 001:01 & 002:01 & Bearded collie & 73 & 27 & 0.67 & $0.40-1.11$ & 0.1355 \\
\hline & & & & WHWT & 1 & 0 & N/A & & \\
\hline 5 & 018:01 & 001:01 & 008:02 & Bearded collie & 70 & 27 & 0.71 & $0.42-1.18$ & 0.2094 \\
\hline \multirow[t]{2}{*}{6} & 015:01 & 006:01 & 022:01 & Bearded collie & 5 & 2 & 0.80 & $0.15-4.17$ & 1 \\
\hline & & & & Labradoodle & 0 & 1 & N/A & & \\
\hline \multirow[t]{3}{*}{7} & 002:01 & 009:01 & 001:01 & Bearded collie & 6 & 0 & N/A & & \\
\hline & & & & Labradoodle & 0 & 1 & N/A & & \\
\hline & & & & Standard poodle & 2 & 0 & N/A & & \\
\hline \multirow[t]{2}{*}{8} & 023:01 & 003:01 & 005:01 & Bearded Collie & 1 & 0 & N/A & & \\
\hline & & & & PWD & 11 & 5 & 2.21 & $0.71-6.84$ & 0.1775 \\
\hline \multirow[t]{5}{*}{9} & 015:02 & 006:01 & 023:01 & Bearded collie & 1 & 0 & N/A & & \\
\hline & & & & Labradoodle & 4 & 3 & 0.50 & $0.10-2.58$ & 0.6786 \\
\hline & & & & Standard poodle & 7 & 8 & 2.26 & $0.78-6.59$ & 0.1586 \\
\hline & & & & PWD & 11 & 5 & 2.21 & $0.71-6.84$ & 0.1775 \\
\hline & & & & WHWT & 4 & 0 & N/A & & \\
\hline 10 & 015:01 & 006:01 & 020:02 & WHWT & 51 & 6 & 0.41 & $0.17-1.00$ & 0.0514 \\
\hline \multirow[t]{3}{*}{11} & 013:01 & 001:01 & 002:01 & Leonberger & 4 & 4 & 1.22 & $0.27-5.59$ & 1 \\
\hline & & & & Standard poodle & 1 & 0 & N/A & & \\
\hline & & & & WHWT & 1 & 0 & N/A & & \\
\hline \multirow[t]{4}{*}{12} & 006:01 & 005:01:1 & 007:01 & Labradoodle & 1 & 2 & 1.55 & $0.13-18.5$ & 1 \\
\hline & & & & Leonberger & 5 & 1 & 0.2 & $0.02-1.86$ & 0.1986 \\
\hline & & & & Standard poodle & 4 & 0 & N/A & & \\
\hline & & & & WHWT & 2 & 0 & N/A & & \\
\hline \multirow[t]{4}{*}{13} & 009:01 & 001:01 & 008:01:1 & Labradoodle & 1 & 3 & 2.43 & $0.23-25.5$ & 0.6227 \\
\hline & & & & Standard poodle & 5 & 2 & 0.72 & $0.14-3.85$ & 1 \\
\hline & & & & WHWT & 3 & 2 & 2.61 & $0.43-15.88$ & 0.5882 \\
\hline & & & & PWD & 8 & 0 & N/A & & \\
\hline \multirow[t]{5}{*}{14} & 001:01 & 001:01 & 002:01 & Labradoodle & 2 & 2 & 0.73 & $0.09-5.72$ & 1 \\
\hline & & & & Leonberger & 11 & 16 & 3.64 & $1.07-12.30$ & 0.0447 \\
\hline & & & & PWD & 57 & 15 & 1.32 & $0.62-2.79$ & 0.5597 \\
\hline & & & & WHWT & 130 & 51 & 2.3 & $1.40-3.67$ & 0.0009 \\
\hline & & & & Standard poodle & 3 & 0 & N/A & & \\
\hline \multirow[t]{3}{*}{15} & 001:01 & 001:01 & 036:01 & Standard poodle & 2 & 0 & N/A & & \\
\hline & & & & WHWT & 6 & 0 & N/A & & \\
\hline & & & & Labradoodle & 0 & 1 & $\mathrm{~N} / \mathrm{A}$ & & \\
\hline
\end{tabular}


Table 5 Frequency, code and odds ratio (OR) for DLA class II three-locus haplotypes observed in the six studied breeds at increased risk for Addison's disease (AD): bearded collie (61 cases, 122 controls), West Highland white terrier (WHWT; 43 cases, 166 controls), standard poodles (30 cases, 55 controls), Portuguese water dog (PWD; 17 cases, 76 controls), Labradoodle (12 cases, 9 controls) and Leonberger (11 cases, 14 controls) (Continued)

\begin{tabular}{|c|c|c|c|c|c|c|c|c|c|}
\hline Code & DRB1 & DQA1 & DQB1 & Breed & $\begin{array}{l}\text { Control } \\
(2 n)\end{array}$ & $\begin{array}{l}\mathrm{AD} \\
(2 n)\end{array}$ & OR & $95 \% \mathrm{Cl}$ & $p$-value ${ }^{\dagger}$ \\
\hline 16 & 001:01 & 009:01 & 001:01 & WHWT & 77 & 14 & 0.64 & $0.34-1.21$ & 0.1886 \\
\hline 17 & 008:02 & 003:01 & 004:01 & PWD & 59 & 9 & 0.57 & $0.25-1.30$ & 0.2373 \\
\hline 18 & 011:01 & 002:01 & 013:03 & PWD & 2 & 0 & N/A & & \\
\hline 19 & 012:01 & 004:01 & 013:03 & PWD & 3 & 0 & N/A & & \\
\hline 20 & 012:01 & 004:01 & 013-017 & Standard poodle & 4 & 0 & N/A & & \\
\hline 21 & 015:01 & 006:01 & 011:01 & WHWT & 25 & 5 & 0.76 & $0.28-2.04$ & 0.6499 \\
\hline 22 & 015:01 & 006:01 & 026:01 & Labradoodle & 1 & 2 & 1.55 & $0.13-18.5$ & 1 \\
\hline \multirow[t]{3}{*}{23} & 015:01 & 009:01 & 001:01 & Labradoodle & 3 & 1 & 0.22 & $0.02-2.29$ & 0.2972 \\
\hline & & & & Standard poodle & 8 & 9 & 2.25 & $0.82-6.18$ & 0.1792 \\
\hline & & & & WHWT & 14 & 6 & 1.70 & $0.63-4.57$ & 0.3926 \\
\hline 24 & 015:02 & 006:01 & 026:01 & Standard poodle & 0 & 1 & N/A & & \\
\hline \multirow[t]{2}{*}{25} & 015:03 & 006:01 & 023:01 & Labradoodle & 2 & 0 & N/A & & \\
\hline & & & & Standard poodle & 4 & 2 & 0.91 & $0.16-5.14$ & 1 \\
\hline 26 & 016:01 & 001:01 & 002:01 & Leonberger & 6 & 1 & 0.16 & $0.02-1.44$ & 0.1064 \\
\hline 27 & 017:01 & 002:01 & 013:03 & WHWT & 1 & 0 & N/A & & \\
\hline \multirow[t]{2}{*}{28} & 020:01 & 004:01 & 013:03 & PWD & 1 & 0 & N/A & & \\
\hline & & & & Standard poodle & 2 & 1 & 0.92 & $0.08-10.3$ & 1 \\
\hline 29 & 084:01 & 009:01 & 001:01 & Labradoodle & 2 & 0 & N/A & & \\
\hline
\end{tabular}

$\mathrm{Cl}$ confidence interval, N/A not enough data points to calculate; ${ }^{\dagger}$ Fisher's exact $\mathrm{p}$-value, significant at $p<0.05$ N.B. DLA-DQB1*013-017 is short hand for DQB1*01303 and 01701 appearing on the same haplotype

Bolded values were statistically significant at $a=0.05$. Haplotype codes $1-9$ are as used in the text, additional codes were added as needed

standard poodles (Additional file 10: Table S10) and Labradoodles (Additional file 11: Table S11). Twenty-nine three-locus haplotypes were identified across the six studied breeds, five of which were shared by more than three breeds (Table 5 - haplotypes 3, 9, 12, 13 and 14). Haplotype 14 was the only one associated with risk for $\mathrm{AD}$ in our studied breeds. We confirm its association with $\mathrm{AD}$ in the WHWT, as previously reported [3], and identify it as the first risk haplotype associated with $\mathrm{AD}$ in the Leonberger. No association was seen for the three other breeds that carried haplotype 14 (Labradoodle, PWD and standard poodle).

\section{SLO in bearded collies}

Whereas DLA-DRB1*018:01 and -DQA1*001:01 were common alleles in the bearded collie, they were present at a much higher frequency among SLO dogs compared to healthy controls with $\mathrm{OR}=9.38\left(p=7.40 \times 10^{-10}\right)$ and $\mathrm{OR}=7.22\left(p=4.19 \times 10^{-7}\right)$, respectively, for SLO disease risk (Additional file 12: Table S12). The majority of SLO dogs $(49 / 50)$ carried a DLA-DQB1*002:01 and/or DLADQB1*008:02 allele although an increased risk for disease was observed only for DLA-DQB1*002:01
$(\mathrm{OR}=2.08, p=0.0029)$. However, both $\mathrm{DQB} 1$ alleles were associated with DLA-DRB1*018:01, and the three-locus haplotypes containing these combinations (haplotypes 4 and 5) were significantly overrepresented among SLO dogs and associated with increased risk for disease (Table 2). While risk for disease was slightly higher for the heterozygous genotype 45 , dogs homozygous for each of the risk haplotypes showed similar risk for disease (Table 3). Despite this, SLO dogs were significantly more homozygous in their DLA-DRB1 $(\mathrm{OR}=8.71 ; 95 \% \mathrm{CI}=$ $\left.3.24-23.43 ; p=1.85 \times 10^{-6}\right)$ and -DQA1 (OR $=6.26$; $95 \%$ $\mathrm{CI}=2.11-18.56 ; p=0.0003$ ) genes than controls (Table 4). Homozygosity across all three loci was also significantly greater among SLO dogs compared to controls $(\mathrm{OR}=$ 2.52; $95 \% \mathrm{CI}=1.26-5.06 ; p=0.0104$ ).

Pairwise genotypic comparisons using logistic regression revealed two major genotype groupings, with genotypes 4 4, 45 and 55 significantly associated with a higher probability for SLO when compared to genotypes 1 4, 2 3, 12 , 3 3, 13 and 11 (Fig. 1b); the inverse of what was seen for AD. Genotypes composed of haplotypes 1 and/or 2 were associated with a reduced probability of having SLO. No sex differences were observed in the analysis $(p>0.05)$. 
When geographical regions were considered in the analysis, haplotype 4 remained a risk haplotype for SLO in Europe, but not in North America (Additional file 13: Table S13). Conversely, haplotype 5 remained a risk for SLO in North America, but not in Europe.

\section{Discussion}

Some of the strongest genetic associations with human autoimmune diseases, such as $\mathrm{AD}$ and type 1 diabetes mellitus, involve MHC class II genes [46]. The present study identified a single DLA class II risk haplotype for $\mathrm{AD}$ in the bearded collie, consistent with a previous report in a smaller number of dogs from the United Kingdom [3], although our data did not support the researchers' observation of haplotype 4 being protective for AD. While highly prevalent among control dogs, this haplotype was also observed at similar proportions in AD bearded collies from our dataset. However, when data from the previously published paper was merged with the newly acquired data, a combined analysis showed that haplotype 4 became slightly underrepresented among cases. This may be due to differences in the geographic origin of the samples. Haplotype 4 was more prevalent among controls of European origin and a risk haplotype for SLO in European bearded collies, but not among those from North America. Conversely, haplotype 5 remained a risk for SLO in dogs sampled from North America, but not those from Europe. This finding could indicate actual geographical differences in susceptibility to disease, although it is more likely that they reflect the significantly reduced sample sizes for SLO cases when the data was split by geographical region, thus reducing our power to detect true associations. As for AD, regional differences in haplotype frequency did not affect our initial observation, and haplotype 1 remained significantly associated with $\mathrm{AD}$ in both Europe and North America.

Surprisingly, the highest risk for $\mathrm{AD}$ was seen when haplotype 1 was combined with the SLO risk haplotype 5 . The sole difference between these two haplotypes is the DLA-DRB1 allele: DLA-DRB1*009:01 and DLADRB1*018:01, respectively. The non-polymorphic DLADRA and polymorphic -DRB1 genes encode two chains (alpha and beta, respectively) of the same MHC class II molecule, whereas the DLA-DQA1 and -DQB1 genes encode chains that combine to form a different MHC class II molecule [47]. MHC molecules actively participate in the positive and negative selection of developing $\mathrm{T}$ cells in the thymus. The recognition of self-peptides associated with MHC molecules results in selection of $\mathrm{T}$ cells that bind $\mathrm{MHC}$ with intermediate-to-low avidity; binding that is too strong represents potential for autoreactivity and results in deletion of such $\mathrm{T}$ cells. Moreover, intermediate avidity, where binding is strong but falls below the threshold for deletion, results in generation of regulatory $\mathrm{T}$ cells capable of suppressing self-antigen presentation in the peripheral lymph nodes, thus preventing autoreactivity [46]. While the exact mechanism through which MHC genes contribute to autoimmune disease development is not fully understood, disease-predisposing MHC molecules appear to confer risk by allowing autoreactive $\mathrm{T}$ cells to escape central tolerance, whereas protective MHC molecules confer resistance to autoimmunity by promoting negative selection and generation of regulatory T cells $[46,47]$. The two DLA-DRB1 alleles mentioned above produce molecules that differ by four amino acids in the hypervariable regions of the gene, which encode the peptide-binding region of an MHC molecule. Amino acid changes in the peptide-binding region of $\mathrm{MHC}$ molecules can affect the repertoire of peptides they are capable of presenting to $\mathrm{T}$ cells [46], which is why MHC heterozygosity is generally associated with increased fitness due to the ability to detect a larger number of pathogenic antigens compared to a homozygous individual $[46,48]$. However, carrying two autoimmune disease-predisposing MHC haplotypes may actually increase the number and types of autoreactive $\mathrm{T}$ cells that escape central tolerance thus increasing the risk of developing autoimmunity. In fact, humans who are heterozygous for the MHC class II risk haplotypes, HLA-DR3 and HLA-DR4, are at much higher risk for autoimmune type 1 diabetes and AD than those with either of the homozygous haplotypes [32, 33, 49]. This may also be the case for bearded collies with $\mathrm{AD}$, where most dogs were heterozygous for the risk haplotype and increased risk was seen in association with the heterozygous 15 genotype.

As expected, considerable diversity of alleles was seen across the six dog breeds at increased risk for AD, but allele sharing was fairly limited. Five of the 29 three-locus haplotypes identified were shared by more than three of the studied breeds (haplotypes 3, 9, 12, 13 and 14), four of which had been previously associated with AD in the cocker spaniel, springer spaniel, WHWT, Labrador retriever, standard poodle and Nova Scotia duck tolling retriever $[3,38]$. However, our study only confirmed association of haplotype 14 with $\mathrm{AD}$ in the WHWT and also identified it as the first risk haplotype associated with $\mathrm{AD}$ in the Leonberger. Haplotype 9, previously associated with $\mathrm{AD}$ in the standard poodle [3], was not confirmed as a risk haplotype for AD in our analysis. Although our dataset had more AD standard poodles, our control group is smaller than the previous study, which may have impaired our ability to detect the association. The absence of an association may also be due to differences between studied populations: our dataset consisted of standard poodles from the United States and United Kingdom, whereas previously published data consisted of dogs almost exclusively from the United Kingdom. 
Two risk haplotypes were identified for SLO in our bearded collies, consistent with previous findings [24] though that study evaluated only Scandinavian bearded collies using many fewer dogs. Furthermore, in our population, almost half of the SLO dogs were homozygous for one of these two risk haplotypes (i.e. genotypes 44 or 5 5). Interestingly, although the highest risk was seen with the heterozygous genotype 45 , dogs homozygous for either of the risk haplotypes appear to be at similar risk for disease. This may be explained by the high similarity between haplotypes 4 and 5 , which differ only in their DLA-DQB1 allele. DLA-DQB1*002:01 (in haplotype 4) differs from DLA-DQB1*008:02 (in haplotype 5) by three neighboring amino acids, only one of which falls within a hypervariable region in exon 2 , as defined by Kennedy et al. in 1999. Since the alleles have similar hypervariable regions, they likely have similar antigen binding and functional properties [50], which could explain why genotypes $4,4,45$, and 55 all offered similar risk for SLO.

Three three-locus haplotypes were associated with reduced risk for expressing SLO: haplotypes 1, 2 and 3. Despite its association with increased risk for AD, haplotype 1 was associated with a lower risk of expressing SLO. In fact, as highlighted above, the sole difference between the SLO risk haplotype 5 and the AD risk haplotype 1 is the DRB1 allele, which may suggest a role for this gene in determining the target tissue for autoimmune disease in the bearded collie. However, strong linkage disequilibrium (LD; non-random association between alleles) in the region may actually be responsible for this observation, and it is possible that tissue-specific genetic determinants exist in nearby genes that are in strong LD with the DLA-DRB1 gene resulting in the observed association.

Perhaps most interesting, however, is that many of the risk haplotypes associated to date with organ-specific autoimmune diseases in dogs carry one of two DQ haplotypes: DLA-DQA1*001:01/DQB1*002:01 (DQ1) or DLA-DQA1*001:01/DQB1*008:02 (DQ2). The DQ1 haplotype is part of haplotype 14, for instance, which has not only been associated with AD in WHWT and Leonbergers, but also with anal furunculosis in the German shepherd [42], hypothyroidism in the Gordon setter [43] and SLO in the giant schnauzer and English setter [24]. Furthermore, the DQ1 combination is found in two different three-locus haplotypes that have been associated with higher risk for hypothyroidism (lymphocytic thyroiditis) in Doberman pinschers, giant schnauzers (DLA-DRB1*012:01/ DQA1*001:01/DQB1*002:01) and English setters (DLADRB1*001:07/DQA1*001:01/DQB1*002:01) [40, 43, 44]. Moreover, the four three-locus DLA class II risk haplotypes associated with SLO in different dog breeds $[24,28,43]$ carry a DQ1 or DQ2 haplotype. These
DQ haplotypes were also observed in two SLO-affected dogs in our database that represent breeds in which SLO is uncommon: one great dane, heterozygous for DQ1, and one Belgian tervuren, homozygous for DQ2 (unpublished finding). DQ2 is found in haplotype 1, the risk haplotype for $\mathrm{AD}$ in the bearded collie and diabetes mellitus in multiple dog breeds [39]. It is also a part of haplotype 5 , which is associated with risk for SLO in the bearded collie and Gordon setter [24, 28, 43], and a three-locus haplotype associated with meningoencephalitis in the greyhound (DLA-DRB1*018:02/ DQA1*001:01/DQB1*008:02) [45]. These observations indicate that DQ haplotypes may constitute signatures of autoimmune predisposition across multiple dog breeds, which perhaps combine with breed-specific genetic components to determine the tissue-specificity of autoimmune disease expression.

Whereas an association between MHC class II haplotypes, particularly DQ haplotypes, and autoimmune disease clearly exists in both dogs and humans, other MHC or non-MHC genetic components likely play a role in autoimmune disease development, which would explain why many dogs carrying the risk haplotype for a disease, such as AD, SLO or anal furunculosis, fail to develop the condition. Although our study points to a strong influence of particular DQ haplotypes in different autoimmune diseases across multiple dog breeds, the fact that a risk haplotype can be associated with a disease in one dog and not another suggests that other loci are necessary to determine disease expression and target organ specificity. In fact, studies in mice have shown that, while MHC genes play a major role in autoimmunity, they are still only a part of the genetic components required for disease development. Studies using nonobese diabetic (NOD) mice, which carry a particular MHC haplotype and are prone to developing diabetes, show that the presence of the diabetes-prone MHC haplotype in a different mouse strain fails to cause diabetes [51]. In contrast, when the diabetes-prone MHC of NOD mice is replaced by a different haplotype, autoimmunity develops but in a different target organ [52], thereby demonstrating tissue-specific susceptibility dependent upon factors additional to the MHC. Therefore, autoimmune disease expression is likely dependent upon the existence of non-MHC genetic determinants cooperating with autoimmune disease-predisposing MHC class II haplotypes.

\section{Conclusion}

Our study results complement published data, and shows that three DLA class II risk haplotypes associated with autoimmune diseases are highly prevalent in the bearded collie population, which may account for incidence of autoimmune disorders in the breed. Moreover, the two-locus DQ haplotypes making up these three risk 
haplotypes are found in other risk haplotypes associated with diabetes mellitus and hypothyroidism across different dog breeds. Multiple studies have clearly demonstrated that DLA class II genes play a role in canine autoimmunity, although associations have been deemed breed- and disease-specific. Our work, in combination with the published literature, revealed common DLA-DQ haplotypes associated with different autoimmune diseases across multiple dog breeds. While additional loci are clearly necessary for actual disease expression, the DQ two-locus model may be a good indicator for susceptibility to certain organ-specific autoimmune diseases in dogs, and future studies on carriers of these risk DQ haplotypes may prove fruitful in identifying the additional genetic components involved in canine autoimmunity. These markers may then be used to make informed breeding decisions with the purpose of reducing the incidence of each disease in the canine population. Furthermore, genetic markers of canine autoimmune diseases may provide insights into their human counterpart.

\section{Methods}

\section{Samples}

Blood or buccal swab samples were obtained from 236 bearded collies (125 healthy, $61 \mathrm{AD}$ and $50 \mathrm{SLO}$ ) in North America, Europe, Australia and New Zealand that were healthy or affected by one of two autoimmune diseases (AD or SLO). Addisonian cases consisted of dogs diagnosed by a veterinarian on the basis of clinical signs, demonstrated electrolyte imbalance (sodium to potassium ratio < 27:1) and confirmed with an ACTH stimulation test. Serum cortisol levels $<2.0 \mu \mathrm{g} / \mathrm{dL}(55 \mathrm{nmol} / \mathrm{L})$ before and after ACTH administration were considered diagnostic of AD. Dogs with a history of corticosteroid use that may have interfered with the ACTH stimulation test were excluded, as well as dogs presenting with atypical AD, characterized by low levels of serum cortisol before and after ACTH administration, but normal electrolyte ratio [19]. SLO cases consisted of dogs diagnosed by a veterinarian through biopsy and/or clinical findings such as pain, abnormal growth of nails, or nails bleeding, splitting or falling off. Control dogs were nine years or older, with no history of autoimmune disease (suspected or diagnosed) and considered healthy based on medical history. Samples were subjected to DNA extraction as previously described [53] and quantified using a Nanodrop ${ }^{\circ}$ spectrophotometer. DNA samples were stored at $-20{ }^{\circ} \mathrm{C}$ until processing. The same procedure was performed for five other dog breeds at increased risk for developing AD: standard poodles (11 cases, 10 controls), WHWT (10 cases, 10 controls), Labradoodles (9 cases, 9 controls), PWD (15 cases, 16 controls) and Leonbergers (11 cases, 13 controls).
To complement the AD data, DLA haplotypes from a previously studied population [3] were included in the analyses for the bearded collie (29 cases, 46 controls from the UK for a total of 90 cases and 168 controls), standard poodle (19 cases, 45 controls from the UK and North America for a total of 30 cases and 55 controls), WHWT (33 cases, 156 controls from the UK for a total of 43 cases and 166 controls), Labradoodle (3 cases from the UK for a total of 12 cases and 9 controls), and PWD ( 2 cases, 60 controls from the USA and UK for a total of 17 cases and 76 controls). The z-ratio test for independent proportions (http://vassarstats.net/propdiff_ind.html) was used to assess differences between geographical regions in the combined dataset [54].

\section{DLA Haplotyping}

Sequence based typing was used to determine DLA haplotypes of all dogs. Amplification and sequencing of exon 2 for each of the three polymorphic MHC class II genes, DLA-DRB1, -DQA1 and -DQB1, were performed using flanking primers as previously described [55-57] and two new primer sets developed for DLA-DRB1 and DQB1 (Additional file 14: Table S14). Sequences from the newly designed primers provided greater coverage upstream and downstream of exon 2, improving sequence quality obtained for the entire exon, and matched bearded collie DQB1 $(n=30)$ and DRB1 $(n=$ 18 ) sequences obtained using published primers. A touchdown polymerase chain reaction (PCR) protocol was initially used for all three genes, which consisted of 14 touchdown cycles with annealing temperatures starting at $62^{\circ} \mathrm{C}$ for DLA-DRB1, $57^{\circ} \mathrm{C}$ for DLA-DQA1 and $73^{\circ} \mathrm{C}$ for DLA-DQB1, and reducing by $0.5^{\circ} \mathrm{C}$ each cycle, and 24 additional cycles with annealing temperatures of $55^{\circ} \mathrm{C}$ for DLA-DRB1, $50{ }^{\circ} \mathrm{C}$ for DLA-DQA1 and $66^{\circ} \mathrm{C}$ for DLA-DQB1. The same touchdown PCR protocol was used with the newly designed DLA-DQB1 primer set, but a standard PCR protocol $\left(95^{\circ} \mathrm{C}\right.$ denaturation, 65 ${ }^{\circ} \mathrm{C}$ annealing, $72{ }^{\circ} \mathrm{C}$ extension, 30 cycles) was used with the new set of DLA-DRB1 primers. Promega GoTaq ${ }^{\circ}$ Flexi DNA Polymerase (Promega, WI, USA) was used for all PCRs in a $25 \mu \mathrm{L}$ reaction. Size and concentration of amplicons were verified by running $5 \mu \mathrm{L}$ of the PCR product on a $1 \%$ agarose gel. PCR products were then purified using Exosap-IT ${ }^{\mathrm{Tm}}$ (Thermo Fisher Scientific, Waltham, MA, USA) according to manufacturer's recommendations, and sequenced by capillary electrophoresis on an Applied Biosystems 3730 (UC Davis DNA Sequencing facility). Nucleotide sequences for each DLA gene were analyzed and alleles assigned using SBTengine v.3.2 (GenDX, Netherlands). Ambiguous sequences were observed in all dog breeds. In these cases, haplotypes were predicted based on the three-locus haplotypes identified within each of the studied populations. If 
alleles could not be determined for one or more of the DLA class II genes, the individual was removed from analysis.

\section{Statistical analysis}

Allele frequencies were calculated for each of the three polymorphic DLA genes. Three-locus DLA-DRB1/DQA1/ DQB1 haplotypes were determined based on individuals that were homozygous on all three loci $(n=63)$, followed by individuals homozygous at two of the loci $(n=87)$. Odds ratio (OR) estimates were also calculated based on the number of cases and healthy controls carrying a particular haplotype compared to the number of individuals not carrying the haplotype. A $2 \times 2$ contingency table was used to calculate the ORs and two-tailed Fisher's exact values for the different haplotypes (http://vassarstats.net/ odds2x2.html). The same approach was used to determine statistical differences in homozygosity of the DLA genes between cases and controls [58]. Statistical significance was considered at $p<0.05$.

Logistic regression was used to model the risk of disease as a function of the observed DLA class II three-locus genotypes. Defining the probability of disease as $p_{i j}$ for a dog identified with DLA haplotype $i$ and DLA haplotype $j$, the logit of this probability was determined as $\theta_{i j}=\log \left[p_{i j} /\left(1-p_{i j}\right)\right]$. Modeling the logit as a function of the genotype used the linear model:

$$
\theta_{i j}=b_{0}+a d d_{i}+a d d_{j}+d o m_{i j}
$$

where $b_{0}$ is an unknown constant common to all dogs, $a d d_{i}$ is the additive contribution of the $i$-th $(i=1,2,3,4,5)$ haplotype to the risk of disease, $a d d_{j}$ is the additive contribution of the $j$-th $(j=1,2,3,4,5)$ haplotype to the risk of disease, and $d m_{i j}$ is the dominance contribution of both haplotypes $i$ and $j$ to the risk of disease. Estimation of the unknown additive and dominance effects, along with providing predictions of the risk of disease, were implemented with the Bayesian statistical package Stan [59] executed with the public domain language $\mathrm{R}$ [60]. The combination of rare haplotypes and a disease of low prevalence, as seen in the data, increased the risk of empty subclasses. A hierarchical Bayesian model with weakly informative prior distributions for the unknown effects stabilized the estimation process [61].

Specifically, the intercept $\left(b_{0}\right)$ was drawn from the prior density Cauchy $(0,10)$, the additive effects $\left(a d d_{i}\right.$ and $\left.a d d_{j}\right)$ were drawn from the prior density $\mathrm{N}\left(0, \sigma_{a}^{2}\right)$, the dominance effects $\left(\operatorname{dom}_{i j}\right)$ were drawn from the prior density $\mathrm{N}\left(0, \sigma_{d}^{2}\right)$, and the standard deviations of the additive and dominance contributions $\left(\sigma_{a}\right.$ and $\left.\sigma_{d}\right)$ were drawn from the positive values of a Cauchy $(0,2.5)$ as recommended for weakly informative priors [62]. The simulation process was conducted across four chains, where each chain was built on a draw of 15,000 total samples, a "burn-in" process of 5000 samples followed by thinning to every 20 -th sample. In this way, each chain generated 500 samples, and 2000 samples across the four chains to generate more stable estimates. Convergence of the process was visualized through trace plots of all the unknown values and computation of the Gelman-Rubin statistic for convergence being below 1.05 [63].

\section{Additional files}

Additional file 1: Table S1. Allele frequency for the three polymorphic DLA class II genes in 233 bearded collies (DOCX 15 kb)

Additional file 2: Table S2. Allele frequency and odds ratio (OR) for Addison's disease (AD; $n=61)$ vs controls $(n=122)$ in bearded collies. Bolded values were statistically significant at $a=0.05$ (DOCX $19 \mathrm{~kb}$ )

Additional file 3: Table S3. Frequency, code, and odds ratio (OR) with 95\% confidence interval (Cl) of each three-locus haplotype observed in healthy, Addisonian (AD) and symmetrical lupoid onychodystrophy (SLO) bearded collies in the combined dataset. Text in bold indicates the haplotype frequency significantly differed between cases and controls. Haplotype codes are as used in Table 5; additional codes added as needed. (DOCX $16 \mathrm{~kb}$ )

Additional file 4: Table S4. Frequency of DLA three-locus haplotypes in European (79 controls, 43 AD and 29 SLO) and North American (80 controls, 45 AD and 19 SLO) bearded collies. Bolded values indicate statistical differences in haplotype frequency according to geographical region as determined by the $z$-ratio test for independent proportions. Haplotype codes are as used in Table 5; additional codes added as needed. (DOCX 16 kb)

Additional file 5: Table S5. Frequency, code, and odds ratio (OR) with 95\% confidence interval (Cl) of each three-locus haplotype observed in healthy and Addisonian (AD) bearded collies by geographical region. Text in bold indicates the haplotype frequency significantly differed between cases and controls. Haplotype codes are as used in Table 5; additional codes added as needed. (DOCX $17 \mathrm{~kb}$ )

Additional file 6: Table S6. Frequency of DLA class II alleles segregating in six dog breeds at higher risk for developing Addison's disease (AD) (DOCX $23 \mathrm{~kb})$

Additional file 7: Table S7. Allele frequency and odds ratio (OR) for Addison's disease (AD; $n=10)$ vs controls $(2 n=10)$ in West Highland white terriers. Bolded values were statistically significant at $a=0.05$ (DOCX $19 \mathrm{~kb}$ )

Additional file 8: Table S8. Allele frequency and odds ratio (OR) for Addison's disease (AD; $n=11)$ vs controls $(n=13)$ in Leonbergers. Bolded values were statistically significant at $a=0.05$ (DOCX $16 \mathrm{~kb}$ )

Additional file 9: Table S9. Allele frequency and odds ratio (OR) for Addison's disease (AD; $n=17)$ vs controls $(n=76)$ in Portuguese water dogs. (DOCX $18 \mathrm{~kb}$ )

Additional file 10: Table S10. Allele frequency and odds ratio (OR) for Addison's disease (AD; $n=30)$ vs controls $(n=55)$ in standard poodles. (DOCX $19 \mathrm{~kb}$ )

Additional file 11: Table S11. Allele frequency and odds ratio (OR) for Addison's disease (AD; $n=12$ ) vs controls $(n=9)$ in Labradoodles. (DOCX $18 \mathrm{~kb})$

Additional file 12: Table S12. Allele frequency and odds ratio (OR) for symmetrical lupoid onychodystrophy (SLO; $n=50)$ vs controls $(n=122)$ in bearded collies. Bolded values were statistically significant at $a=0.05$. (DOCX $19 \mathrm{~kb}$ )

Additional file 13: Table S13. Frequency, code, and odds ratio (OR) with $95 \%$ confidence interval (CI) of each three-locus haplotype observed in healthy and symmetrical lupoid onychodystrophy (SLO) bearded collies by geographical region. Text in bold indicates the haplotype frequency 
significantly differed between cases and controls. Haplotype codes are as used in Table 5; additional codes added as needed. (DOCX $16 \mathrm{~kb}$ )

Additional file 14: Table S14. Primer sequences used for DLA class II haplotyping (DOCX $13 \mathrm{~kb}$ )

\section{Acknowledgements}

We gratefully acknowledge the infrastructure support of the Department of Animal Science, College of Agricultural and Environmental Sciences, and the California Agricultural Experiment Station of the University of California, Davis. The authors would also like to thank the Bearded Collie Foundation for Health, Dr. Elsa Sell and Dr. Linda Aronson for their invaluable help in bearded collie sample recruitment; Jo Tucker (Canine Immune Mediated Disease Awareness, CIMDA) for being instrumental in encouraging sample collection; the technical staff supporting this study, Maria Palij, Jenifer Hallock, Sini Karjalainen, Reetta Hänninen, Kaisu Hiltunen, and Steven Quarmby; Ezinne Ibe, and Simon Rothwell at the UK DNA Archive for Companion Animals; and all the owners and clinicians who have actively participated in our research projects as well as every dog that contributed to the study. We would also like to thank IDEXX laboratories (Harrogate, UK), Nationwide Laboratories (Poulton-le-Fylde, UK), Angela Pedder, Colleen Stead, the Bearded Collie Breed Club (UK Northern Branch), Lucy Davison and Betty Aughey.

\section{Funding}

This study was funded in part by BeaCon (Bearded Collie Foundation for Health) the American Kennel Club Canine Health Foundation (AMO; 02187-MOU and $\# 1236-A)$, the Academy of Finland, Helsinki Institute of Life Science and the Jane and Aatos Erkko Foundation, and the European Commission FP7 project number 201167, Euradrenal.

\section{Availability of data and materials}

All data generated or analyzed during this study are included in this published article and its supplementary information files (Supplemental Tables).

\section{Authors' contributions}

LCG and AMO designed the study. All authors collected samples. LCG and JMB genotyped the samples with assistance from ML and AS. LCG and TRF conducted the statistical analyses. $L C G, J M B$, and AMO interpreted the findings and drafted the manuscript. LJK and $\mathrm{HL}$ contributed to data interpretation, and LJK provided study input and significantly edited the manuscript. All authors read and approved the final manuscript.

\section{Ethics approval and consent to participate}

All applicable international, national, and/or institutional guidelines for the care and use of animals were followed. All procedures performed were in accordance with the ethical standards of the University of California, Davis (IACUC \#20402) and University of Helsinki, Finland (permit ESAVI/6054/ 04.10.03/ 2012).

All UK samples consisted of residual blood remaining after diagnostic testing and were collected in accordance with guidelines of the Royal College of Veterinary Surgeons, UK and the Veterinary Surgeons Act 1966. For this reason ethical committee approval was not required. All samples were collected with informed and written owner consent.

\section{Consent for publication}

Not applicable.

\section{Competing interests}

Lorna Kennedy is Managing Editor on Canine Genetics and Epidemiology. The other authors declare no competing interests.

\section{Publisher's Note}

Springer Nature remains neutral with regard to jurisdictional claims in published maps and institutional affiliations.

\section{Author details}

'Department of Animal Science, University of California, One Shields Ave, Davis, CA 95616, USA. ${ }^{2}$ Brazilian National Council for Scientific and
Technological Development (CNPq) fellow, Brasília, Brazil. ${ }^{3}$ Centre for Integrated Genomic Medical Research, University of Manchester, Manchester, UK. ${ }^{4}$ Research Programs Unit, Molecular Neurology, and Department of Veterinary Biosciences, University of Helsinki, Helsinki, Finland. ${ }^{5}$ Folkhälsan Institute of Genetics, Helsinki, Finland.

Received: 10 December 2018 Accepted: 24 January 2019

Published online: 15 February 2019

\section{References}

1. Boag AM, Catchpole B. A review of the genetics of Hypoadrenocorticism. Topics in Companion Animal Medicine. 2014;29(4):96-101.

2. Boag AM, Christie MR, McLaughlin KA, Syme HM, Graham P, Catchpole B. Autoantibodies against cytochrome P450 side-chain cleavage enzyme in dogs (Canis lupus familiaris) affected with Hypoadrenocorticism (Addison's disease). PLoS One. 2015;10(11):e0143458.

3. Massey J, Boag A, Short AD, Scholey RA, Henthorn PS, Littman MP, Husebye E, Catchpole B, Pedersen N, Mellersh CS, et al. MHC class II association study in eight breeds of dog with hypoadrenocorticism. Immunogenetics. 2013; 65(4):291-7.

4. Short AD, Boag A, Catchpole B, Kennedy LJ, Massey J, Rothwell S, Husebye E, Ollier B. A candidate gene analysis of canine Hypoadrenocorticism in 3 dog breeds. J Hered. 2013;104(6):807-20.

5. Charmandari E, Nicolaides NC, Chrousos GP. Adrenal insufficiency. Lancet. 2014;383(9935):2152-67.

6. Mitchell AL, Pearce SHS. Autoimmune Addison disease: pathophysiology and genetic complexity. Nat Rev Endocrinol. 2012;8(5):306-16.

7. Oberbauer AM, Benemann KS, Belanger JM, Wagner DR, Ward JH, Famula TR. Inheritance of hypoadrenocorticism in bearded collies. Am J Vet Res. 2002:63(5):643-7.

8. Soderbergh A, Winqvist O, Norheim I, Rorsman F, Husebye ES, Dolva O, Karlsson FA, Kampe O. Adrenal autoantibodies and organ-specific autoimmunity in patients with Addison's disease. Clin Endocrinol. 1996:45(4):453-60.

9. Falorni A, Nikoshkov A, Laureti S, Grenback E, Hulting AL, Casucci G, Santeusanio F, Brunetti P, Luthman H, Lernmark A. High diagnostic accuracy for idiopathic Addison's disease with a sensitive radiobinding assay for autoantibodies against recombinant human 21-hydroxylase. J Clin Endocrinol Metab. 1995;80(9):2752-5.

10. Oberbauer A, Bell J, Belanger J, Famula T. Genetic evaluation of Addison's disease in the Portuguese water dog. BMC Vet Res. 2006;2(1):15.

11. Online Mendelian Inheritance in Animals, OMIA. Sydney School of Veterinary Science, 12/05/2018. World Wide Web URL: https://omia.org/ home/. Accessed 05 Dec 2018.

12. Decôme M, Blais M-C. Prevalence and clinical features of hypoadrenocorticism in great Pyrenees dogs in a referred population: 11 cases. The Canadian veterinary journal $=$ La revue veterinaire canadienne. 2017:58(10):1093-9.

13. Bellumori TP, Famula TR, Bannasch DL, Belanger JM, Oberbauer AM. Prevalence of inherited disorders among mixed-breed and purebred dogs: 27,254 cases (1995-2010). J Am Vet Med Assoc. 2013;242(11):1549-55.

14. Hanson JM, Tengvall K, Bonnett BN, Hedhammar A. Naturally occurring adrenocortical insufficiency--an epidemiological study based on a Swedishinsured dog population of 525,028 dogs. J Vet Intern Med. 2016;30(1):76-84.

15. Wiles BM, Llewellyn-Zaidi AM, Evans KM, O'Neill DG, Lewis TW. Large-scale survey to estimate the prevalence of disorders for 192 kennel Club registered breeds. Canine genetics and epidemiology. 2017;4:8.

16. Famula TR, Belanger JM, Oberbauer AM. Heritability and complex segregation analysis of hypoadrenocorticism in the standard poodle. J Small Anim Pract. 2003:44(1):8-12.

17. Hughes AM, Nelson RW, Famula TR, Bannasch DL. Clinical features and heritability of hypoadrenocorticism in Nova Scotia duck tolling retrievers: 25 cases (1994-2006). J Am Vet Med Assoc. 2007;231(3):407-12.

18. Burkitt JM. Chapter 76 - Hypoadrenocorticism. In: Silverstein DC, Hopper K, editors. Small Animal Critical Care Medicine. Saint Louis: W.B. Saunders; 2009. p. 321-4.

19. Klein SC, Peterson ME. Canine hypoadrenocorticism: part I, vol. 51; 2010

20. Van Lanen K, Sande A. Canine Hypoadrenocorticism: pathogenesis, diagnosis, and treatment. Topics in Companion Animal Med. 2014;29(4):88-95.

21. Auxilia ST, Hill PB, Thoday KL. Canine symmetrical lupoid onychodystrophy: a retrospective study with particular reference to management. J Small Anim Pract. 2001;42(2):82-7. 
22. Mueller RS, Rosychuk RA, Jonas LD. A retrospective study regarding the treatment of lupoid onychodystrophy in 30 dogs and literature review. Am Anim Hosp Assoc. 2003;39:139-50.

23. Scott D, Rousselle S Jr. MW: symmetrical lupoid onychodystrophy in dogs: a retrospective analysis of 18 cases (1989-1993). J Am Anim Hosp Assoc. 1995; 31(3):194-201.

24. Wilbe M, Ziener ML, Aronsson A, Harlos C, Sundberg K, Norberg E, Andersson L, Lindblad-Toh K, Hedhammar Å, Andersson G. DLA class II alleles are associated with risk for canine symmetrical lupoid onychodystropy (SLO). PLoS One. 2010;5(8):e12332.

25. Ziener ML, Nødtvedt A. A treatment study of canine symmetrical onychomadesis (symmetrical lupoid onychodystrophy) comparing fish oil and cyclosporine supplementation in addition to a diet rich in omega-3 fatty acids. Acta Vet Scand. 2014;56(1):66.

26. Ziener ML, Bettenay SV, Mueller RS. Symmetrical onychomadesis in Norwegian Gordon and English setters. Vet Dermatol. 2008;19(2):88-94.

27. Scott DW, Miller WH. Disorders of the claw and claw bed in dogs. Compendium on Continued Education for the Practicing Veterinarian. 1992;14(11):12.

28. Dahlgren S, Ziener ML, Lingaas F. A genome-wide association study identifies a region strongly associated with symmetrical onychomadesis on chromosome 12 in dogs. Anim Genet. 2016;47(6):708-16.

29. The Bearded Collie Foundation for Health. BeaCon Open Health Registry Report. The Bearded Collie Foundation for Health. 2017. https://www. beaconforhealth.org/YR_17_OHR_Report.pdf. Accessed June 162018.

30. Rosenblum MD, Remedios KA, Abbas AK. Mechanisms of human autoimmunity. J Clin Invest. 2015;125(6):2228-33.

31. Rioux JD, Abbas AK. Paths to understanding the genetic basis of autoimmune disease. Nature. 2005;435(7042):584-9.

32. Myhre AG, Undlien DE, Løvås K, Uhlving S, Nedrebø BG, Fougner KJ, Trovik T, Sørheim Jl, Husebye ES. Autoimmune adrenocortical failure in Norway autoantibodies and human leukocyte antigen class II associations related to clinical features. J Clin Endocrinol Metab. 2002;87(2):618-23.

33. Noble JA, Valdes AM. Genetics of the HLA region in the prediction of type 1 diabetes. Current Diab Rep. 2011:11(6):533-42.

34. Stokkers PCF, Reitsma PH, Tytgat GNJ, van Deventer SJH. HLA-DR and -DQ phenotypes in inflammatory bowel disease: a meta-analysis. Gut. 1999;45(3):395.

35. Catchpole B, Adams JP, Holder AL, Short AD, Ollier WER, Kennedy LJ. Genetics of canine diabetes mellitus: are the diabetes susceptibility genes identified in humans involved in breed susceptibility to diabetes mellitus in dogs? Vet J. 2013;195(2):139-47.

36. Dyggve H, Kennedy LJ, Meri S, Spillmann T, Lohi H, Speeti M. Association of Doberman hepatitis to canine major histocompatibility complex II. Tissue Antigens. 2011;77(1):30-5.

37. Greer KA, Wong AK, Liu H, Famula TR, Pedersen NC, Ruhe A, Wallace M, Neff MW. Necrotizing meningoencephalitis of pug dogs associates with dog leukocyte antigen class II and resembles acute variant forms of multiple sclerosis. Tissue Antigens. 2010;76(2):110-8.

38. Hughes AM, Jokinen P, Bannasch DL, Lohi H, Oberbauer AM. Association of a dog leukocyte antigen class II haplotype with hypoadrenocorticism in Nova Scotia duck tolling retrievers. Tissue Antigens. 2010;75(6):684-90.

39. Kennedy $L$, Davison $L$, Barnes A, Short AD, Fretwell N, Jones CA, Lee AC, Ollier WER, Catchpole B. Identification of susceptibility and protective major histocompatibility complex haplotypes in canine diabetes mellitus. Tissue Antigens. 2006;68(6):467-76.

40. Kennedy LJ, Huson HJ, Leonard J, Angles JM, Fox LE, Wojciechowski JW, Yuncker C, Happ GM. Association of hypothyroid disease in Doberman pinscher dogs with a rare major histocompatibility complex DLA class II haplotype. Tissue Antigens. 2006;67(1):53-6.

41. Kennedy LJ, Ollier WER, Marti E, Wagner JL, Storb RF. Canine Immunogenetics. In: Ostrander EA, Ruvinsky A, editors. The Genetics of the Dog. 2nd ed. London: CAB International; 2012. p. 91-126.

42. Kennedy LJ, O'Neill T, House A, Barnes A, Kyostila K, Innes J, Fretwell N, Day MJ, Catchpole B, Lohi H, et al. Risk of anal furunculosis in German shepherd dogs is associated with the major histocompatibility complex. Tissue Antigens. 2008;71(1):51-6.

43. Ziener ML, Dahlgren S, Thoresen SI, Lingaas F. Genetics and epidemiology of hypothyroidism and symmetrical onychomadesis in the Gordon setter and the English setter. Canine genetics and epidemiology. 2015;2(1):12.

44. Wilbe M, Sundberg K, Hansen IR, Strandberg E, Nachreiner RF, Hedhammar A, Kennedy $\sqcup$, Andersson G, Bjornerfeldt S. Increased genetic risk or protection for canine autoimmune lymphocytic thyroiditis in Giant schnauzers depends on DLA class II genotype. Tissue Antigens. 2010;75:712-9.

45. Shiel RE, Kennedy $\sqcup$, Nolan CM, Mooney CT, Callanan JJ. Major histocompatibility complex class II alleles and haplotypes associated with non-suppurative meningoencephalitis in greyhounds. Tissue Antigens. 2014;84(3):271-6.

46. Tsai S, Santamaria P. MHC Class II Polymorphisms, Autoreactive T-Cells, and Autoimmunity. Front Immunol. 2013;4:321.

47. Holzer U, Nepom GT. Major Histocompatibility Complex and Autoimmune Disease. In: Burt RK, Marmont AM, editors. Stem Cell Therapy for Autoimmune Disease. Georgetown: Landes Bioscience; 2004. p. 155-62.

48. Piertney SB, Oliver MK. The evolutionary ecology of the major histocompatibility complex. Heredity. 2005;96:7.

49. Skanes VM, Barnard J, Farid N, Marshall WH, Murphy L, Rideout D, Taylor R, Xidos G, Larsen B. Class III alleles and high-risk MHC haplotypes in type diabetes mellitus, Graves' disease and Hashimoto's thyroiditis. Molecular biology \& medicine. 1986;3(2):143-57.

50. Kennedy LJ, Altet L, Angles JM, Barnes A, Carter SD, Francino O, Gerlach JA, Happ GM, Ollier WE, Polvi A, et al. Nomenclature for factors of the dog major histocompatibility system (DLA), 1998. First report of the ISAG DLA nomenclature committee. Int Society for Animals Genetics Tissue Antigens. 1999:54(3):312-21.

51. Wicker LS, Todd JA, Peterson LB. Genetic control of autoimmune diabetes in the NOD mouse. Annu Rev Immunol. 1995;13:179-200.

52. Braley-Mullen H, Sharp GC, Medling B, Tang H. Spontaneous autoimmune thyroiditis in NOD.H-2h4 mice. J Autoimmun. 1999;12(3):157-65.

53. Rincon G, Tengvall K, Belanger JM, Lagoutte L, Medrano JF, Andre C, Thomas A, Lawley CT, Hansen MS, Lindblad-Toh K, et al. Comparison of buccal and blood-derived canine DNA, either native or whole genome amplified, for arraybased genome-wide association studies. BMC Res Notes. 2011;4:226.

54. Davies-Mostert HT, Mills MGL, Macdonald DW: Hard boundaries influence African wild dogs' diet and prey selection. 2013, 50(6):1358-1366.

55. Bexfield NH, Watson PJ, Aguirre-Hernandez J, Sargan DR, Tiley L, Heeney JL, Kennedy LJ. DLA class II alleles and haplotypes are associated with risk for and protection from chronic hepatitis in the English springer spaniel. PLoS One. 2012;7(8):e42584.

56. Kennedy LJ, Barnes A, Happ GM, Quinnell RJ, Courtenay O, Carter SD, Ollier WER, Thomson W. Evidence for extensive DLA polymorphism in different dog populations. Tissue Antigens. 2002;60(1):43-52.

57. Wagner JL, Burnett RC, Works JD, Storb R. Molecular analysis of DLA-DRBB1 polymorphism. Tissue Antigens. 1996;48(5):554-61.

58. Kim HY. Statistical notes for clinical researchers: chi-squared test and Fisher's exact test. Restorative dentistry \& endodontics. 2017:42(2):152-5.

59. Carpenter B, Gelman A, Hoffman MD, Lee D, Goodrich B, Betancourt M, Brubaker M, Guo J, Li P, Riddell A: Stan: a probabilistic programming language. 2017 2017, 76(1):32\%J Stat Softw.

60. R Core. Team: R: a language and environment for statistical computing. In: R Foundation for statistical Computing; 2017.

61. Gelman A, Carlin J, Stern H, Dunson D, Vehtari A, Rubin D. Bayesian data analysis, third edition (chapman \& \{hall/CRC $\}$ texts in statistical science). In: Chapman and Hall/CRC; 2013.

62. Gelman AJBa: Prior distributions for variance parameters in hierarchical models (comment on article by Browne and Draper). 2006, 1(3):515-534.

63. Gelman A, Rubin DBJSs: Inference from iterative simulation using multiple sequences. 1992, 7(4):457-472.

Ready to submit your research? Choose BMC and benefit from:

- fast, convenient online submission

- thorough peer review by experienced researchers in your field

- rapid publication on acceptance

- support for research data, including large and complex data types

- gold Open Access which fosters wider collaboration and increased citations

- maximum visibility for your research: over $100 \mathrm{M}$ website views per year

At $\mathrm{BMC}$, research is always in progress.

Learn more biomedcentral.com/submissions 